\title{
THE SIZE OF BOSE-EINSTEIN EFFECTS IN THE STATISTICAL BOOTSTRAP MODEL
}

\author{
J. ENGELS \\ Department of Theor. Physics, University' of Bielefeld, Germany \\ and \\ K. FABRICIUS and K. SCHILLING \\ Faculty of Physics, University of Wuppertal, Germany
}

Received 3 October 1975

\begin{abstract}
We have determined the shift in Hagedorn temperature due to Bose statistics and calculated explicitly the cluster expansions of fireball level densities. This enabled us to investigate Bose effects in one-particle spectra and integrated correlations. Apart from the low secondary energy region of the one-particle distributions, the nonasymptotic behaviour of these quantities is little affected.
\end{abstract}

Introduction. In a recent paper, Hagedorn and collaborators [1] have derived a cluster decomposition for the density of states of an ideal relativistic gas with Bose-Einstein or Fermi-Dirac statistics, from which they conclude that Boltzmann statistics expressions provide a poor approximation in all situations where a microcanonical description is needed. This raises the question about the actual size of effects due to correct statistics in the statistical bootstrap model (SBM) as formulated by Frautschi [2]. Although the bootstrap equation with quantum statistics and the analytic structure of its solution has been given by $\mathrm{Nahm}$ [3] and was later on discussed in ref. [4], all detailed nonasymptotic investigations about fireball decay performed so far started from the Boltzmann version of the SBM [5-7], mainly because it is much easier to handle [8].

In view of the intriguing possibility that SBM might provide a picture to understand $\mathrm{e}^{+} \mathrm{e}^{-}$annihilation into hadrons and could thus be tested in rather great detail by experiments at SPEAR, DORIS and subsequent machines [7], we have for the first time solved the full bootstrap equation [3] for identical pions and a truncated bootstrap equation (that allows a fireball to decay into two fireballs only) for pions with charge, both cases with correct Bose-Einstein statistics.

In this paper we want to give a brief account on the consequences of quantum statistics for fireball decay. In particular we show the mean multiplicities, in- tegrated correlations and the one-particle spectrum.

Solution of the full bootstrap. The full bootstrap equation for the grand canonical partition function of a system of identical bosons of mass $m$ reads [3]

$2 Z(\beta)=t_{1}(\beta, m)+\sum_{n=1}^{\infty} \frac{1}{n !}\left(\sum_{k=1}^{\infty} \frac{Z(k \cdot \beta)}{k}\right)^{n}$.

Here

$$
\begin{aligned}
& Z(\beta)=\int \mathrm{d}^{4} Q \exp (-\beta Q) \tau\left(Q^{2}\right), \\
& t_{i}(\beta, m)=B \int \mathrm{d}^{4} Q \exp (-\beta Q) \delta\left(Q^{2}-(\mathrm{i} m)^{2}\right) \theta\left(Q_{\mathrm{o}}\right), \\
& \quad i=1,2, \ldots .
\end{aligned}
$$

The contributions $k>1$ are just due to the Bose counting; the Boltzmann case amounts to taking the first term in the $k$-sum only.

Eq. (1) can be rewritten in a form suitable for iteration

$Z(\beta)=Z_{\mathrm{y}}\left[t_{1}(\beta, m)+2 R(\beta)\right]-R(\beta)$,

with

$R(\beta)=\sum_{k=2}^{\infty} \frac{Z(k \cdot \beta)}{k}$

Here $Z_{\mathrm{y}}$ is the solution of the bootstrap equation for 
the Boltzmann case. A sequence of functions $Z^{(N)}$ is defined through

$$
Z^{(N)}(\beta)=Z_{\mathrm{y}}\left[t_{1}(\beta, m)+2 R^{(N-1)}(\beta)\right]-R^{(N-1)}(\beta),
$$

with

$R^{(N)}(\beta)=\sum_{k=2}^{\infty} \frac{Z^{(N)}(k \cdot \beta)}{k}, \quad R^{(0)}(\beta) \equiv 0$,

which converges to the solution of eq. (1). This iteration process has been proposed, but not exploited, in ref. [4].

The solution of the problem proceeds in two steps:

i) determination of the ultimate temperature $T_{\mathrm{H}}$ $=\beta_{\mathrm{H}}^{-1}$ in terms of the volume parameter $B$,

ii) calculation of the cluster expansion for $Z$.

We have used eq. (5) together with the temperature equation

$t_{1}\left(\beta_{\mathrm{H}}^{(N+1)}, m\right)=t_{1}\left(\beta_{\mathrm{H}}^{(0)}, m\right)-2 R^{(N)}\left(\beta_{\mathrm{H}}^{(N+1)}\right)$

thus obtaining a sequence $\beta^{(N)}$ that converges to the inverse ultimate temperature $T_{\mathrm{H}}^{-1}$. The corresponding quantity for the Boltzmann case is denoted by $\beta_{\mathrm{H}}^{(0)}$. The computer calculation yields for $B=3.145 \mathrm{GeV}^{-2}$ a Hagedorn temperature $T_{\mathrm{H}}^{(0)}=169.7 \mathrm{MeV}$ for the Boltzmann and $T_{\mathrm{H}}=160.0 \mathrm{MeV}$ for the Bose cases. In the following, we have chosen in both cases the same value of $160 \mathrm{MeV}$ for the ultimate temperature, which requires $B=3.655 \mathrm{GeV}^{-2}$ for Boltzmann statistics.

Secondly, we want to obtain the coefficients $h$ of the cluster expansion of $Z$ :

$Z(\beta)=\sum_{k=1}^{\infty} \sum_{n_{1}, \ldots, n_{k}=0}^{\infty} h\left(n_{1}, \ldots, n_{k}\right) \prod_{i=1}^{k} t_{i}^{n_{i}}(\beta, m)$.

This can be achieved by inserting the Yellin expansion for $Z_{\mathrm{y}}$

$Z_{\mathrm{y}}(s)=\sum_{n=1}^{\infty} g_{n} s^{n}$

into eq. (5) and utilizing the identity

$t_{i}(k \cdot \beta, m)=\frac{t}{k^{2}} t_{i \cdot k}(\beta, m)$.

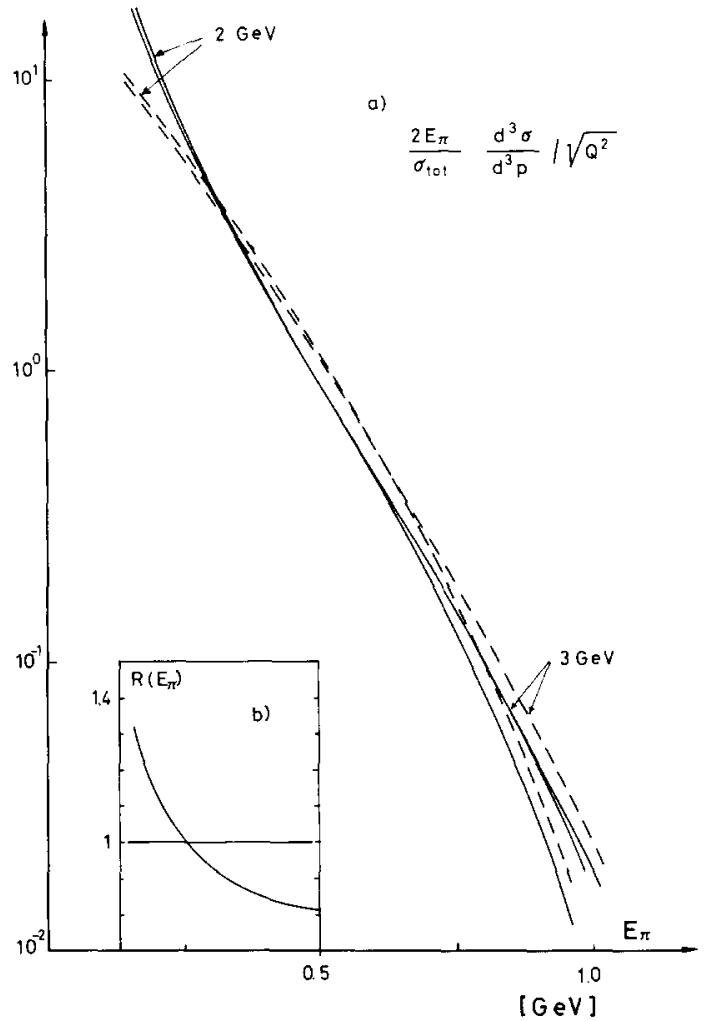

Fig. 1a) Normalized one-particle spectra divided by $\sqrt{Q^{2}}$ for $\sqrt{Q^{2}}=2$ and $3 \mathrm{GeV}$ in the full bootstrap model. The curves for Bose statistics (full lines) and Boltzmann statistics (broken lines) were both calculated with the same ultimate temperature $T_{\mathrm{H}}=T_{\mathrm{H}}^{(0)}=160 \mathrm{MeV}$. b) $R\left(E_{\pi}\right)=$ Bose $/$ Boltzmann distribution multiplied by $\left[1-\exp \left(-E_{\pi} / 160 \mathrm{MeV}\right)\right]$ at $\sqrt{Q^{2}}$ $=3 \mathrm{GeV}$.

We observe that the $(N+1)$ st iteration step does not change the coefficients belonging to particle numbers $n=\sum_{i=1}^{k} i \cdot n_{i}$ up to $n=N$. Therefore the iteration process can be considerably simplified by discarding on the r.h.s. of eq. (5) all terms with $n>N$. Yet, this procedure leads to a myriad of terms: We managed to calculate all coefficients up to $n=16$ which amounts to 914 terms instead of 16 for the Boltzmann case.

Once the $h$ 's are known, the calculation of inclusive quantities for firebal, decay is straightforward: by inverse Laplace transformation, the partition function $Z(\beta)$ goes over into the fireball level density for mass $\sqrt{Q^{2}}$. 


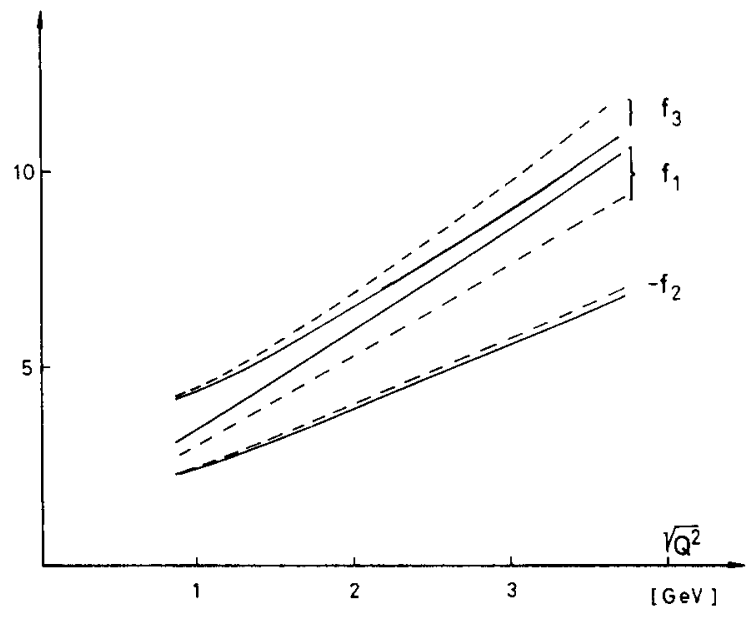

Fig. 2. The correlation functions $f_{1}, f_{2}, f_{3}$ in the full bootstrap model; parameters and notation as in fig. 1 a.

$$
\begin{gathered}
\tau\left(Q^{2}\right)=\sum_{k=1}^{\infty} \sum_{n_{1}, \ldots, n_{k}=0}^{\infty} h\left(n_{1}, \ldots, n_{k}\right) \\
\times B^{l-1} \Omega^{l}(Q^{2} ; \underbrace{m, \ldots, m}_{n_{1} \text { times }}, \ldots, \underbrace{k m, \ldots, k m}_{n_{k} \text { times }})
\end{gathered}
$$

where $\Omega^{l}$ is the Lorentz-invariant phase space for $l$ clusters, $l=\sum_{i=1}^{k} n_{i}$. The inclusive one-particle spectrum is given by

$$
\begin{aligned}
& \frac{2 p_{\mathrm{o}}}{\sigma_{\text {tot }}} \frac{\mathrm{d}^{3} \sigma}{\mathrm{d}^{3} p}=\tau^{-1}\left(Q^{2}\right) \sum_{k=1}^{\infty} \sum_{n_{1}, \ldots, n_{k}=0}^{\infty} h\left(n_{1}, \ldots, n_{k}\right) \\
& \times B^{l-1} \sum_{j=1}^{k} n_{j} j^{3} \Omega^{l-1}\left((Q-j p)^{2}, \ldots\right),
\end{aligned}
$$

where one $j$-cluster is taken away in $\Omega^{l-1}$ with respect to $\Omega^{l}$.

In fig. 1 we show the normalized one-particle spectrum which we divided by $\sqrt{Q^{2}}$ in view of the fact that $\bar{n}$ grows asymptotically like $\sqrt{Q^{2}}$. Since the 2 and $3 \mathrm{GeV}$ curves almost coincide for smaller secondary energies $E_{\pi}=p_{0}$ we conclude that asymptotia is reached at these energies. The Boltzmann curve is proportional to $\exp \left(-E_{\pi} / T_{\mathrm{H}}^{(0)}\right)$ in this region. Bose effects are expected to be strongest near $E_{\pi}=m$, and there is in fact a remarkable enhancement of the Bose over the Boltzmann distribution for $E_{\pi} \lesssim 300 \mathrm{MeV}$. The enhancement differs however from the free-gas Bose spectrum which behaves as $\left[\exp \left(E_{\pi} / T_{\mathrm{H}}\right)-1\right]^{-1}$ : this is demonstrated by the variation of the quantity $R\left(E_{\pi}\right)$, which is defined as the ratio of the Bose/ Boltzmann distribution multiplied by $\left[1-\exp \left(-E_{\pi}\right]\right.$ $160 \mathrm{MeV})]$ and plotted in fig. 1. It is obvious that the enhancement leads to an increase in the average multiplicity $f_{1}$ which is plotted in fig. 2 , together with the integrated correlations $f_{2}, f_{3}$.

Solution of the quadratic bootstrap. Since the charge problem seems unmanageable within the full bootstrap for Bose statistics, we simplify the problem to the quadratic bootstrap which is the simplest situation allowing Bose effects. The equation for the isoscalar and isovector partition functions $S$ and $Z_{\mathrm{I}_{3}}$ read:

$S=\frac{1}{6}\left\{Z_{\mathrm{o}}^{2}+2 Z_{+} Z_{-}\right\}+\frac{1}{2}\left\{S(2 \beta)+S^{2}\right\}$,

$Z_{ \pm}=t_{1, \pm}+Z_{ \pm}\left(\frac{1}{2} Z_{\mathrm{o}}+S\right)$,

$Z_{\mathrm{o}}=t_{1,0}+\frac{1}{2} Z_{+} Z_{-}+Z_{0} S$.

All functions are understood to be of argument $\beta$ unless stated otherwise. The charge of the pion inhomogeneity $t_{1}$ has been indicated. A term proportional to $Z_{\mathrm{o}}(2 \beta)$ is not allowed in eq. (12a) because $Z_{\mathrm{o}}(2 \beta)$ does not behave like an isoscalar. As $S(2 \beta)$ describes two fireballs in the same state its Clebsch-Gordon-coefficient should be the same as that of $S^{2}$. The technique to solve eq. (12) by iteration closely resembles the procedure applied in the full bootstrap case.

As a result we find for $B=2.9215 \mathrm{GeV}^{-2}$ the value $T_{\mathrm{H}}^{(0)}=160.05 \mathrm{MeV}$ for the Boltzmann and $T_{\mathrm{H}}=160$ $\mathrm{MeV}$ for the Bose case. In the calculations we shifted $T_{\mathrm{H}}^{(0)}$ to $T_{\mathrm{H}}^{(0)}=160 \mathrm{MeV}$, corresponding to $B=2.9240$ $\mathrm{GeV}^{-2}$. For each of the four partition functions, one needs more than 400 terms in the cluster expansions up to 16 pions. As expected from the small temperature shift, the cluster effects are small as well in the other quantities, such as the integrated correlations. The ratio $\left(f_{\mathrm{BOSE}}-f_{\mathrm{BOLTZMANN}}\right) / f_{\mathrm{BOSE}}$ is of the order of $10^{-4}$. So the speculation that the "energy crisis" observed in $\mathrm{e}^{+} \mathrm{e}^{-}$annihilation [9] might be due to Bose effects [10] turns out to be wrong at least within the quadratic bootstrap scheme.

We conclude that Bose effects do not considerably alter the predictions of SBM with Boltzmann statistics. An exception is the inclusive spectrum at small secondary energies. The influence of Bose statistics on differ- 
ential two particle correlations will be investigated within the SBM in a following paper.

\section{References}

(1) M. Chaichian, R. Hagedorn and M. Hayashi, CERN preprint TH 1975, 1975.

[2] S. Frautschi, Phys. Rev. D3 (1971) 2821.

[3] W. Nahm, Nucl. Phys. B45 (1972) 525.

[4] K. Fabricius and U.M. Wambach, Nucl. Phys. B62 (1973) 212.

[5] J. Yellin, Nucl. Phys. B52 (1973) 583.

[6] R. Hagedorn and I. Montvay, Nucl. Phys. B59 (1973) 45; E.M. Ilgenfritz and J. Kripfganz, Nucl. Phys. B62 (1973) 141 .
[7] J. Engels, H. Satz and K. Schilling, Nuovo Cim. 17A (1973) 535;

J. Engels, K. Fabricius and K. Schilling, Nuovo Cim. 23A (1974) 581 ;

J. Engels, K. Fabricius and K. Schilling, Phys. Lett. 53B (1974) 65.

[8] See the review given by I. Montvay, Budapest preprint KFKI-75-43.

[9] B. Richter, rapporteur's talk at the XVII Int. Conf. on High energy physics, London 1974.

[10] U.M. Wambach, Dortmund preprint, The results on the abundance of neutrals in this paper must be wrong. This can already be seen from the fact that there the isoscalar fireball leads to $f_{+}$different from $f_{0}$. This might be due to an incorrect volume dependence given in ref. [4]. 